\title{
Risk factors for repetition and suicide following self-harm in older adults: multicentre cohort study
}

Elizabeth Murphy, Navneet Kapur, Roger Webb, Nitin Purandare, Keith Hawton, Helen Bergen, Keith Waters and Jayne Cooper

\section{Background}

Older adults have elevated suicide rates. Self-harm is the most important risk factor for suicide. There are few population-based studies of self-harm in older adults.

\section{Aims}

To calculate self-harm rates, risk factors for repetition and rates of suicide following self-harm in adults aged 60 years and over.

\section{Method}

We studied a prospective, population-based self-harm cohort presenting to six general hospitals in three cities in England during 2000 to 2007.

\section{Results}

In total 1177 older adults presented with self-harm and
$12.8 \%$ repeated self-harm within 12 months. Independent risk factors for repetition were previous self-harm, previous psychiatric treatment and age 60-74 years. Following selfharm, 1.5\% died by suicide within 12 months. The risk of suicide was 67 times that of older adults in the general population. Men aged 75 years and above had the highest suicide rates.

\section{Conclusions}

Older adults presenting to hospital with self-harm are a high-risk group for subsequent suicide, particularly older men.

\section{Declaration of interest}

None.
Self-harm is the most important risk factor for suicide, and the risk increases markedly with age. ${ }^{1}$ Older adults have high suicide rates in many countries. ${ }^{2}$ Increases in suicide rates with advancing age were seen in approximately half of countries examined in a recent World Health Organization (WHO) study. ${ }^{3}$ In the UK older adult suicide rates have declined in recent years, therefore rates are currently highest in younger age groups. ${ }^{4}$ However, the rate ratio of self-harm to completed suicide converges from greater than 30 in all ages to less than 10 among those aged 60 years and over. ${ }^{5}$ It has therefore been suggested that self-harm in older age more closely resembles suicide, and involves greater suicidal intent and lethality of method. ${ }^{6}$ Despite the elevated risk of suicide in older adults who self-harm, there are few large prospective population-based studies of this group. ${ }^{7}$ Few studies have examined risk factors for repetition of self-harm or for suicide, and these have mostly been conducted in small and selective samples. ${ }^{8,9}$ One previous study did include a large sample of older adults and a 20-year follow-up period, but was limited to one centre. ${ }^{10}$ Also, in applying long observation periods to maximise statistical power, strong temporal effects may be overlooked in pooled analyses across the whole study period. ${ }^{11}$

We conducted a large population-based study of patients presenting with self-harm in three centres in England. ${ }^{12}$ The objectives of the study were to examine older adults presenting to hospital with self-harm with respect to age- and gender-specific rates of self-harm, risk factors for repetition of self-harm and rates of suicide following self-harm.

\section{Method}

\section{Design and setting}

The Multicentre Monitoring of Self Harm Project is a prospective cohort study of self-harm presentations to emergency departments

†See editorial, pp. 356-358, this issue. at six general hospitals in three centres in England: Oxford (one hospital), Manchester (three hospitals) and Derby (two hospitals). ${ }^{12}$ The city of Manchester is an urban area with a population of 381236 ; Derby unitary area comprises an urban area surrounded by rural districts and has a population of 204 032; and Oxford city contains rural areas both within and around the city and has a population of $128411 .{ }^{13}$ According to the UK government's Index of Multiple Deprivation, in which 353 local authority areas in England were given an overall deprivation score, Manchester was ranked the 4th most deprived, Derby was ranked 69th and Oxford was ranked 156th. ${ }^{14}$

\section{Ethical considerations}

Oxford and Derby both have approval from local National Health Service (NHS) research ethics committees to collect data on selfharm for local monitoring and multicentre projects. The monitoring of self-harm in Manchester is part of a clinical audit system and has been ratified as such by local research ethics committees. Thus, formal ethics committee approval was not required for that centre. All centres are fully compliant with the UK Data Protection Act 1998, and have support under section 251 of the NHS Act 2006 regarding the use of patient-identifiable information.

\section{Data collection}

We defined older adults as individuals aged 60 years and above. This is consistent with previous UK studies of self-harm among older adults, ${ }^{10,15}$ as well as in other countries. ${ }^{16}$ We used established monitoring methods to identify consecutive episodes of self-harm presenting to the study hospitals, as described in full elsewhere. ${ }^{17,18}$ Self-harm was defined as intentional self-poisoning or self-injury, irrespective of motivation and degree of suicidal intent, ${ }^{19}$ and this was applied consistently across all three centres. Demographic data and details on method of self-harm were 
collected from emergency department records for all episodes. Most participants received a psychosocial assessment from emergency department staff and/or mental health specialists. Clinicians recorded sociodemographic data, method of self-harm, precipitating factors and other clinical information. The latter included self-reported previous self-harm, drug or alcohol misuse and psychiatric treatment (including antidepressant treatment by a general practitioner as well as psychiatric out-patient or in-patient treatment). For participants who were not assessed by clinicians (for example, because they refused or took early discharge), basic information was collected by research clerks from medical records.

Self-harm repetition was defined as a second presentation with non-fatal self-harm to any of the hospitals within each study centre during the study period. Identification of repeat self-harm involved a comprehensive data linkage process at each centre based on individual identifiers. Suicide following self-harm was defined as deaths by suicide (ICD-10 codes X60-X84) and undetermined cause (Y10-Y34, excluding Y33.9), hereafter referred to as 'suicides. ${ }^{20}$ Verdicts of undetermined cause are conventionally included in research on suicide and in official suicide statistics, ${ }^{21}$ and the exclusion of ICD-10 code Y33.9 refers to cases where the coroner's verdict was still pending. A comprehensive matching process was used to identify cases of suicide from national death registers across the UK through the Medical Research Information Service of the NHS. The matching process also revealed whether participants had died from causes other than suicide, or had left national health registers (for example, owing to emigration) at the end of follow-up (see Appendix).

\section{Statistical analysis}

Self-harm data were analysed for an 8-year study period for Oxford and Derby (1 January 2000 to 31 December 2007). For Manchester, data were unavailable for non-assessed episodes prior to September 2002. Therefore, Manchester self-harm data were analysed for a period of 5 years and 4 months (1 September 2002 to 31 December 2007), when data were available for both assessed and non-assessed individuals. The study period for identification of deaths began at the start of the self-harm observation period at each centre, until 31 December 2008, thus allowing an additional 12 months of mortality follow-up. The analyses were conducted using SPSS version 16.0 for Windows and Intercooled Stata version 10. Analysis was based on an individual's first presentation of self-harm at the age of 60 years or over. First, self-harm rates per 100000 person-years were calculated for individuals aged at least 60 years and with a postcode within the city catchment area of each of the hospitals in Manchester, Derby and Oxford. Population estimates were obtained for each city for the individual years 2000 to 2007 from the Office for National Statistics. ${ }^{13}$ We chose an upper age band of 75 years or over as used in previous studies of suicidal behaviour. $^{4,22}$ Approximate person-years at risk were generated by multiplying gender- and age-specific population estimates for each catchment area by the applicable study period for that centre. Mortality rate ratios categorised by gender and age group were calculated using Poisson regression models, with no significant evidence of overdispersion. Exact 95\% confidence intervals were calculated for the rate ratios.

Second, we examined repetition of self-harm as an outcome. The risk of repetition within 12 months was calculated (excluding individuals with fewer than 365 days of follow-up during the study period). Potential risk factors for repetition were investigated using hazard ratios (HRs) generated by Cox's proportional hazards models. Patients were censored at the default study exit date, or earlier in case of death or emigration. Univariate associations were examined initially, and then domain-specific multivariate models were generated using backwards elimination procedures (separate models for domains of sociodemographic factors, circumstances of self-harm, clinical factors and precipitants). Explanatory variables were retained in these models if $P$ was less than 0.2 . The independent predictors from all the domain-specific models were then fitted in a final multivariate model. Explanatory variables were retained in the final model if $P$ was less than 0.05 .

Third, we examined suicide as an outcome following selfharm. Suicide rates following self-harm per 100000 person-years at risk were calculated. Poisson regression was used to examine suicide rates categorised by gender and age group (with no significant evidence of overdispersion) and exact $95 \%$ confidence intervals were calculated for the mortality rate ratios. We calculated age- and gender-standardised mortality ratios to compare the number of suicides in the self-harm cohort with the expected number of suicides in the general population. Indirect standardisation was used to adjust for age (stratified in 10-year bands) and gender differences between the study population and that of England and Wales from 2000 to 2007. A standardised mortality ratio (SMR) for younger adults aged 20-59 years was generated for comparison with the older adult SMR. We also calculated the risk of suicide within 12 months of self-harm among older adults aged 60 years and over (excluding individuals with fewer than 365 days of follow-up owing to death from other causes or leaving national health registers). Finally, potential risk factors for suicide were examined using Cox's proportional hazard models.

\section{Results}

A total of 21774 adults aged at least 20 years presented with selfharm over the study period. The study cohort consisted of 1177 individuals who presented with self-harm at age 60 years or over (Derby $n=421$, Manchester $n=404$, Oxford $n=352$ ). The maximum age was 97 years (median 68 years, interquartile range $63-77)$ and $56 \%$ of the cohort were female. The most common method of harm used by older adults was self-poisoning alone $(88 \%)$, followed by self-injury including cutting $(9 \%)$ and violent methods such as hanging or asphyxiation (3\%). Women were more likely to self-poison $(93 \% v .81 \%, P<0.001)$ and men were more likely to self-injure $(13 \% v .6 \%, P<0.001)$ and use violent methods ( $7 \%$ v. $1 \%, P<0.001)$. Among men, those over 75 years old were most likely to use violent methods $(10 \%$ v. $5 \%$ aged 60-74 years, $P<0.01)$. Following self-harm, $81 \%$ received a psychosocial assessment and $75 \%$ were admitted to a medical ward. Aftercare referrals were made for $90 \%$, including out-patient care (36\%), psychiatric in-patient care (19\%) and general practitioner care alone $(36 \%)$.

\section{Rates of self-harm}

The rate of self-harm for individuals aged 60 years and over was 65 per 100000 in both men and women (Derby 62, Manchester 69 and Oxford 64 per 100000 ). This compares with a rate of 380 per 100000 in younger adults aged 20-59 years. Table 1 shows rate ratios for self-harm in older adults by age group, for all centres combined. Among women, rates were significantly lower for those aged 75 years and over $v$. those aged 60-74 years. Rates of self-harm among older men did not differ significantly by age group. We tested for differences between centres in the rate ratios using a Wald chi-squared test for heterogeneity. The rate ratios did not vary significantly by centre for women $\left(\chi^{2}=0.81, P=0.37\right)$. 


\begin{tabular}{|c|c|c|c|c|}
\hline & $\begin{array}{c}\text { Self-harm } \\
n\end{array}$ & $\begin{array}{l}\text { Person- } \\
\text { years }\end{array}$ & $\begin{array}{l}\text { Rate/ } \\
100000\end{array}$ & $\begin{array}{c}\text { RR } \\
(95 \% \mathrm{Cl})\end{array}$ \\
\hline \multicolumn{5}{|l|}{ Men } \\
\hline 60-74 years & 185 & 275633 & 67 & 1.00 \\
\hline $75+$ years & 83 & 133800 & 62 & $0.92(0.70-1.20)$ \\
\hline \multicolumn{5}{|l|}{ Women } \\
\hline $60-74$ years & 234 & 294333 & 80 & 1.00 \\
\hline $75+$ years & 102 & 220033 & 46 & $0.58(0.46-0.74)$ \\
\hline
\end{tabular}

Among men the heterogeneity in the rate ratios was not statistically significant $\left(\chi^{2}=3.11, P=0.08\right)$.

\section{Repetition of self-harm}

A total of 196 individuals (16.7\%) repeated self-harm before the end of the study period. The incidence of repeat self-harm within 12 months was $12.8 \%$ (95\% CI 10.6-14.9) (117 of 917 individuals, excluding those with fewer than 365 days of follow-up). Predictors of repetition of self-harm are shown in Tables 2 and 3. Significant sociodemographic predictors in the univariate model included: age 60-74 years; not being married or partnered; and not living with partner, relative or friend (Table 2). Clinical predictors

Table 2 Risk factors for self-harm repetition:

sociodemographic characteristics and circumstances of self-harm $(n=1177)$

\begin{tabular}{|c|c|c|c|}
\hline & $n$ & $\begin{array}{c}\text { Repeated } \\
\text { self-harm } \\
n(\%)\end{array}$ & $\begin{array}{c}\text { Hazard ratio } \\
\text { (univariate) } \\
\text { HR (95\% Cl) }\end{array}$ \\
\hline \multicolumn{4}{|l|}{ Sociodemographic characteristics ${ }^{a}$} \\
\hline \multicolumn{4}{|l|}{ Age, years } \\
\hline $75+$ & 395 & $33(8)$ & 1.0 \\
\hline $60-74$ & 782 & $163(21)$ & $2.4(1.6-3.5)^{*}$ \\
\hline \multicolumn{4}{|l|}{ Gender } \\
\hline Female & 516 & $80(16)$ & 1.0 \\
\hline Male & 661 & $116(17)$ & $1.1(0.8-1.4)$ \\
\hline \multicolumn{4}{|l|}{ Ethnicity } \\
\hline White & 859 & $152(18)$ & 1.0 \\
\hline Other ethnic group & 16 & $2(13)$ & $0.7(0.2-2.8)$ \\
\hline \multicolumn{4}{|l|}{ Marital status } \\
\hline Married, partnered & 244 & $41(17)$ & 1.0 \\
\hline Single, divorced or widowed & 367 & $82(22)$ & $1.5(1.0-2.1)^{\star}$ \\
\hline \multicolumn{4}{|l|}{ Living arrangements } \\
\hline \multicolumn{4}{|l|}{ Spouse/partner, relative } \\
\hline Alone & 244 & $54(22)$ & $1.5(1.0-2.3)^{*}$ \\
\hline \multicolumn{3}{|l|}{ Other (hostel, institution, } & $2.3(1.2-4.2)^{\star}$ \\
\hline \multicolumn{4}{|l|}{ Circumstances of self-harm } \\
\hline Self-poisoning only & 1031 & $178(17)$ & 1.0 \\
\hline $\begin{array}{l}\text { Self-injury (cut, stab, } \\
\text { other injury) }\end{array}$ & 107 & $13(12)$ & $0.7(0.4-1.3)$ \\
\hline Violent method (e.g. hanging) & 39 & $5(13)$ & $0.7(0.3-1.8)$ \\
\hline \multicolumn{4}{|l|}{ Alcohol use at time of harm } \\
\hline No & 574 & $88(15)$ & 1.0 \\
\hline Yes & 343 & $72(21)$ & $1.3(0.96-1.8)$ \\
\hline $\begin{array}{l}{ }^{*} P<0.05 \text {. } \\
\text { a. The level of data completeness fo } \\
100 \% \text {, with the exception of marital }\end{array}$ & , & ving & $\begin{array}{l}\text { rom } 70 \% \text { to } \\
\text { hents }(48 \%) \text {. }\end{array}$ \\
\hline
\end{tabular}

included: current psychiatric treatment; previous psychiatric treatment; previous self-harm; and alcohol or drug problems as a precipitating factor for self-harm (Table 3 ). In the multivariate model, independent predictors of repetition were age 60-74 years v. 75 years and over $(\mathrm{HR}=1.8,95 \%$ CI $1.2-2.8)$, previous psychiatric treatment $(\mathrm{HR}=1.8,95 \%$ CI $1.2-2.7)$ and previous self-harm $(\mathrm{HR}=1.9,95 \%$ CI $1.4-2.8)$. We repeated the analysis using a cut-off of 65 years and the results were similar: previous self-harm $(\mathrm{HR}=3.0,95 \%$ CI 1.8-4.9) and age less than 75 years $(\mathrm{HR}=1.7,95 \% \mathrm{CI} 1.0-2.7)$ remained as independent risk factors.

\section{Suicide following self-harm}

In total, 24 of the 1177 individuals (2.0\%) subsequently died by suicide, of whom 14 were men and 10 were women. The majority of suicides occurred soon after self-harm; $50 \%$ died by suicide within 6 months, $67 \%$ within 12 months and $83 \%$ within 18 months. The incidence of suicide within 1 year of the self-harm episode was $1.5 \%$ (95\% CI $0.8-2.2$ ) (16 out of 1064, allowing all individuals 365 days of follow-up). Considering the final methods used by the 24 individuals who died by suicide, the majority involved violent means (58\%, including hanging, drowning, jumping and firearms) followed by self-poisoning $(25 \%)$ and self-injury (17\%). Violent methods of suicide were used more often by men ( $79 \%$ v. 30\%, $P=0.04$, Fisher's exact test). Prior to suicide, the most common method of self-harm used in the index episode was self-poisoning $(75 \% ; n=18)$, following which $50 \%$ switched to a violent method in the final act. We also examined univariate predictors of suicide following self-harm. The only significant risk factor was the use of a violent

Table 3 Risk factors for self-harm repetition: clinical

characteristics and precipitants $(n=1177)$

\begin{tabular}{|c|c|c|c|}
\hline & $n$ & $\begin{array}{c}\text { Repeated } \\
\text { self-harm } \\
n(\%)\end{array}$ & $\begin{array}{l}\text { Hazard ratio } \\
\text { (univariate) } \\
\text { HR }(95 \% \mathrm{Cl})\end{array}$ \\
\hline \multicolumn{4}{|c|}{ Clinical characteristics ${ }^{a}$} \\
\hline \multicolumn{4}{|c|}{ Current psychiatric treatment } \\
\hline No & 506 & $77(15)$ & 1.0 \\
\hline Yes & 416 & $85(20)$ & $1.4(1.0-1.9)^{*}$ \\
\hline \multicolumn{4}{|c|}{ Previous psychiatric treatment } \\
\hline No & 396 & $41(10)$ & 1.0 \\
\hline Yes & 497 & $119(24)$ & $2.3(1.6-3.3)^{*}$ \\
\hline \multicolumn{4}{|c|}{ Previous self-harm } \\
\hline No & 481 & $53(11)$ & 1.0 \\
\hline Yes & 353 & $97(27)$ & $2.5(1.8-3.6)^{*}$ \\
\hline \multicolumn{4}{|c|}{ Precipitants $^{\mathrm{a}}$} \\
\hline \multicolumn{4}{|c|}{ Physical health problem } \\
\hline No & 582 & $111(19)$ & 1.0 \\
\hline Yes & 376 & $58(15)$ & $0.9(0.6-1.2)$ \\
\hline \multicolumn{4}{|c|}{ Relationship problem } \\
\hline No & 630 & $110(17)$ & 1.0 \\
\hline Yes & 333 & $58(17)$ & $0.9(0.6-1.3)$ \\
\hline \multicolumn{4}{|c|}{ Mental health problem } \\
\hline No & 679 & $118(17)$ & 1.0 \\
\hline Yes & 290 & $52(18)$ & $1.1(0.8-1.5)$ \\
\hline \multicolumn{4}{|c|}{ Bereavement } \\
\hline No & 815 & $146(18)$ & 1.0 \\
\hline Yes & 136 & $22(16)$ & $0.9(0.6-1.5)$ \\
\hline \multicolumn{4}{|c|}{ Alcohol problem } \\
\hline No & 727 & $113(16)$ & 1.0 \\
\hline Yes & 104 & $29(28)$ & $1.9(1.3-2.9)^{*}$ \\
\hline \multicolumn{4}{|c|}{ Drug problem } \\
\hline No & 823 & $139(17)$ & 1.0 \\
\hline Yes & 4 & $3(75)$ & $4.8(1.5-15.1)^{*}$ \\
\hline
\end{tabular}


method in the index episode ( $\mathrm{HR}=4.3,95 \%$ CI $1.3-14.4)$. This was based on 3 of 34 individuals (9\%) who self-harmed with a violent method. All three were male, and they subsequently also used a violent method in the final act.

\section{Standardised mortality ratios}

The number of suicides in the self-harm cohort was 67 times higher (95\% CI 45-99) than the expected number of deaths based on age- and gender-equivalent suicide rates in the general population (Table 4). This compares with an SMR of $24(95 \%$ CI 21-27) among younger adults aged 20-59 years. The SMR among older adults was 2.8 times higher (95\% CI 1.8-4.2) than in younger adults.

\section{Suicide rates categorised by gender and age}

The rate of suicide within the self-harm cohort was higher in men than in women (Table 4), but the difference did not reach statistical significance (rate ratio $\mathrm{RR}=2.0,95 \% \mathrm{CI} 0.8-4.9$ ). When the cohort was stratified by age group, there was no difference between female $v$. male rates of suicide for those aged 60-74 years $(\mathrm{RR}=1.1,95 \%$ CI $0.3-3.7)$. For women, 9 of 10 suicides were among those aged $60-74$ years, a rate of 520 per 100000 . A reliable rate could not be calculated for women aged 75 years or over. However, of the 14 male suicides, 8 were among those aged 75 years and above, a rate of 1624 per 100000 . This was significantly higher than the suicide rate of 477 per 100000 in men aged 60-74 years $(\mathrm{RR}=3.4,95 \%$ CI 1.0-11.9).

\section{Discussion}

We found that the risk of suicide was 67 times higher among older adults presenting to hospital with self-harm relative to the general population. The relative risk in older adults was almost three times greater than the relative risk among younger patients presenting with self-harm. Men aged 75 years and over were at highest risk of suicide following self-harm. Use of a violent method of harm may also be a risk factor for subsequent suicide. Independent risk factors for non-fatal repetition of self-harm were age 60-74 years, previous self-harm and previous psychiatric treatment.

\section{Strengths and limitations}

The main strength of this study is its population-based prospective cohort design, based on large cohorts from three centres, using a recent follow-up period reflective of contemporary services. We used a comprehensive tracing process to identify all suicides in the UK. We were able to censor cases of people who died from causes other than suicide or who left the country, although we were unable to identify suicides among individuals

\begin{tabular}{|c|c|c|c|c|}
\hline & $\begin{array}{c}\text { Suicide } \\
n\end{array}$ & $\begin{array}{l}\text { Person- } \\
\text { years }^{a}\end{array}$ & $\begin{array}{c}\text { Rate/ } \\
100000\end{array}$ & $\begin{array}{c}\text { SMR } \\
(95 \% \mathrm{Cl})\end{array}$ \\
\hline All individuals & 24 & 4198 & 572 & 67 (45-99) \\
\hline Men & 14 & 1749 & 800 & $60(36-102)$ \\
\hline Women & 10 & 2449 & 408 & $78(42-146)$ \\
\hline \multicolumn{5}{|c|}{$\begin{array}{l}\text { SMR, standardised mortality ratio. } \\
\text { a. Based on the total sum of years between each person's index self-harm episode } \\
\text { until date of death, or end date of the study period if living, or date of leaving } \\
\text { national health registers (e.g. owing to emigration). }\end{array}$} \\
\hline
\end{tabular}

who emigrated. We defined older adults as individuals aged 60 years and over. There is no standard criterion for defining older age, although the ages of 60 or 65 years are often used. ${ }^{23}$ We chose 60 years as consistent with previous studies. ${ }^{10,15,16}$ In the UK entry into older adult psychiatric services begins at 65 years. However, most self-harm presentations occur outside normal office hours, ${ }^{17}$ and these people are likely to be seen by on-call junior doctors in psychiatry or liaison mental health nurses. When the analysis was repeated using a cut-off of 65 years, the results for risk factors for repetition were similar to the primary analysis using the cut-off of 60 years.

Despite the large size of our cohort, the relatively small number of suicides affected the power of the study. We were therefore unable to comprehensively examine risk factors for completed suicide. However, this limitation was also observed in the only previous cohort study to examine suicide following self-harm in older adults. ${ }^{10}$ There were some missing data on clinical predictors of repetition for individuals who did not receive a psychosocial assessment, which may be a source of bias. However, non-assessed individuals did not differ in terms of age and gender. Finally, our data on non-fatal repeated self-harm were based on hospital presentations. We did not collect data on repeat self-harm in the community. We were interested in hospital presentations because of their impact on resource use and because repeated hospital presentation for self-harm is associated with suicide. ${ }^{11}$

\section{Findings in relation to previous studies}

The overall self-harm rate of 65 per 100000 is consistent with a previous study conducted in several European countries. ${ }^{2}$ Further breakdown by age showed a decrease in self-harm among the oldest women. Thus, men aged 75 years and over had higher rates than their female counterparts. This mirrors previous findings that the high female to male ratio of self-harm seen in younger adulthood converges and reverses in older age. ${ }^{24}$ This may reflect different motivations for self-harm; for example, a decline in the use of self-harm as a coping strategy, ${ }^{24}$ and a closer resemblance between self-harm and suicide among older adults. ${ }^{6}$

Although the overall incidence of self-harm in older adults was lower than in younger groups, the risk of suicide following selfharm was markedly raised. The SMR in this study was somewhat higher than previously reported. ${ }^{10}$ However, there were methodological differences, including a 20 -year study period in a single centre, in which cohort effects may have been operating. In addition, we observed that rates of suicide following self-harm were strikingly raised among men aged 75 years and over, which has not been previously reported. This may reflect increasing suicidal intent with increasing old age, ${ }^{25,26}$ as well as greater use of violent methods of self-harm among the oldest men, as observed in our study. Furthermore, medical complications are common following self-harm in older adults, ${ }^{27}$ and the oldest individuals may be even less likely to survive the injuries resulting from a repeat attempt. Conversely, among women most suicides occurred in the 60-74 year age group. This is consistent with previous findings that women in a 'young old' group aged 60-69 years used self-harm methods of greater lethality than those over 70 years old, which is the reverse of the trend seen in men. ${ }^{28}$

The rate of repetition of self-harm was somewhat lower than that observed in adults of all ages. ${ }^{29,30}$ This has been reported in previous studies of older adults. ${ }^{8,10,25}$ Factors associated with repetition, such as personality disorder and drug and alcohol misuse, are less common among older adults. ${ }^{6,8}$ Furthermore, repetition in older adults may be carried out for different motivations, i.e. to end life rather than for other reasons. ${ }^{6}$ We also 
found that individuals aged 75 years and over were at lower risk of repetition compared with those aged $60-74$ years. This may be due to the oldest individuals having higher mortality rates due to suicide or other causes, ${ }^{10}$ rather than re-presenting with non-fatal self-harm.

Although there are few prospective studies of self-harm repetition in older adults, the risk factors were consistent with those found in adults of all ages, particularly the importance of previous self-harm. ${ }^{29}$ Contact with psychiatric services has also been associated with repetition among older adults, ${ }^{8}$ and such factors are useful for informing risk assessment. In the univariate analysis we also identified the social risk factors of living alone and not having a partner, and clinical risk factors of alcohol and substance misuse. These have been highlighted as risk factors for suicidal behaviour in case-control studies. ${ }^{31}$ Although the latter factors were not independent predictors for repetition in our study, they are potentially modifiable; for example, interventions that improve social contact, support and integration in the community, ${ }^{32}$ or that treat alcohol misuse, might be of benefit.

Finally, with respect to predictors of suicide, we identified violent method of self-harm as being a possible risk factor. However, this finding should be interpreted cautiously, as it was based on only three cases of suicide. Previous studies have not identified violent method as a risk factor in older adults, most probably owing to underpowered sample sizes. However, violent method has been identified as a predictor of suicide in a recent national Swedish cohort study, which had greater power to examine this effect. ${ }^{33}$

\section{Implications of the study}

Older adults presenting to hospital with self-harm are a high-risk group for subsequent suicide, and emergency departments may therefore be a key setting in terms of their potential for suicide prevention. All older adults presenting with self-harm should be considered as being at elevated risk of suicide unless detailed psychosocial assessment indicates otherwise. In particular, men over 75 years old need to be carefully monitored and assessed, as the risk of suicide is particularly increased in this group. These findings emphasise the requirement for all older adults to receive a detailed psychosocial assessment by a mental health practitioner experienced in the assessment of older people, in accordance with national guidance. ${ }^{34,35}$ Elizabeth Murphy, BSC, Navneet Kapur, FRCPsych, Roger Webb, PhD,
Nitin Purandare, FRCPsych, Centre for Mental Health and Risk, University of Manchester; Keith Hawton, FRCPsych, Helen Bergen, PhD, Centre for Suicide Research, University of Oxford; Keith Waters, RMN, Mental Health Liaison Team, Rehabilitation Centre, Royal Derby Hospital, Derbyshire; Jayne Cooper, PhD, Centre for Mental Health and Risk, University of Manchester, UK

Correspondence: Ms Elizabeth Murphy, Centre for Mental Health and Risk, University of Manchester, Jean McFarlane Building, Oxford Road, Manchester M13 9PL, UK. Email: elizabeth.murphy@manchester.ac.uk

First received 12 Mar 2011, final revision 22 Jul 2011, accepted 15 Sep 2011

\section{Funding}

We acknowledge financial support from the UK Department of Health under the Nationa Health Service Research and Development programme (DH/DSH2008). K.H. is a National Institute for Health Research senior investigator.

\section{Acknowledgements}

The authors thank their respective research teams, and clinical and administrative staff in oxford, Manchester and Derby, for their assistance with self-harm data collection.

\section{Appendix}

\section{Identification of self-harm repetition}

Repeat presentations of self-harm to emergency departments at the six general hospitals within the study centres were identified by linking individuals on name and date of birth. For cases where there was any doubt, additional identifiers were used (hospital number and postcode of residence). The hospitals in each study centre covered a geographically defined city catchment area, and there was no other emergency department within the city where adults could have presented. Outside the cities of Oxford and Derby there was no other hospital within the immediate vicinity. For Manchester, we did not have a direct estimate of how many self-harm episodes may have presented to hospitals in the surrounding districts. However, a recent audit of all hospitals within a $24 \mathrm{~km}$ radius suggested that less that $5 \%$ of all attendances by Manchester residents occurred at hospitals outside the study area.

\section{Identification of suicide cases}

Details of all individuals - name, gender, date of birth, postcode of residence and National Health Service (NHS) number - were submitted to the Medical Research Information Service of the NHS for matching with national death registers. Cases were matched automatically in the first instance. For cases where a single corresponding match was not returned, manual searching was carried out to identify the matching patient entry. Matching revealed whether individuals were alive or dead, including the date and cause of death if applicable, or whether an individual had left the register for other reasons (such as emigration). A successful match was returned for $98 \%$ of adults aged 20 years and over, and for all adults aged 60 years and over.

\section{References}

1 Hawton K, Zahl D, Weatherall R. Suicide following deliberate self-harm: long-term follow-up of patients who presented to a general hospital. Br J Psychiatry 2003; 182: 537-42.

2 De Leo D, Padoani W, Scocco P, Lie D, Bille-Brahe U, Arensman E, et al Attempted and completed suicide in older subjects: results from the WHO/ EURO Multicentre study of suicidal behaviour. Int J Geriatr Psychiatry 2001; 16: $300-10$.

3 Shah A. The relationship between suicide rates and age: an analysis of multinational data from the World Health Organization. Int Psychogeriatr 2007; 19: 1141-52.

4 Shah A, Bhat R, Mackenzie S, Koen C. Elderly suicide rates: cross-national comparisons of trends over a 10-year period. Int Psychogeriatr 2008; 20 : 673-86.

5 Hawton $\mathrm{K}$, Harriss L. How often does deliberate self-harm occur relative to each suicide? A study of variations by gender and age. Suicide Life Threat Behav 2008; 38: 650-60

6 Draper B. Attempted suicide in old age. Int J Geriatr Psychiatry 1996; 11 577-87.

7 Chan J, Draper B, Banerjee S. Deliberate self-harm in older adults: a review of the literature from 1995 to 2004. Int J Geriatr Psychiatry 2007; 22: 720-32.

8 Hepple J, Quinton C. One hundred cases of attempted suicide in the elderly. Br J Psychiatry 1997; 171: 42-6.

9 De Leo D, Padoani W, Lonnqvist J, Kerkhof A, Bille-Brahe U, Michel K, et al. Repetition of suicidal behaviour in elderly Europeans: a prospective longitudinal study. J Affect Disord 2002; 72: 291-5.

10 Hawton K, Harriss L. Deliberate self-harm in people aged 60 years and over: characteristics and outcome of a 20-year cohort. Int J Geriatr Psychiatry 2006; 21: 572-81.

11 Cooper J, Kapur N, Webb R, Lawlor M, Guthrie E, Mackway-Jones K, et al. Suicide after deliberate self-harm: a 4-year cohort study. Am J Psychiatry 2005; 162: 297-303

12 Bergen $\mathrm{H}$, Hawton K, Waters K, Cooper J, Kapur N. Epidemiology and trends in non-fatal self-harm in three centres in England: 2000-2007. Br J Psychiatry 2010; 197: 493-8.

13 Office for National Statistics. Mid Year Population Estimates 2000 to 2007. ONS, 2007 (http://www.ons.gov.uk/ons/taxonomy/ index.html?nscl=Population+Estimates). 
14 Department for Communities and Local Government. Indices of Deprivation. Department for Communities and Local Government, 2007 (http:// www.communities.gov.uk/documents/communities/pdf/733520.pdf).

15 Shah A. Attempted suicide in the elderly in England: age-associated rates, time trends and methods. Int Psychogeriatr 2009; 21: 889-95.

16 Lawrence D, Almeida OP, Hulse GK, Jablensky AV, Holman CDJ. Suicide and attempted suicide among older adults in Western Australia. Psychol Med 2000; 30: 813-21.

17 Hawton K, Bergen H, Casey D, Simkin S, Palmer B, Cooper J, et al. Self-harm in England: a tale of three cities - multicentre study of self-harm. Soc Psychiatry Psychiatr Epidemiol 2007; 42: 513-21.

18 Waters K, Stalker C. 2007 Annual Report of Both Reported Self Harm and Mental Health Presentation, Presenting to the Derby Acute Hospitals. Derbyshire Mental Health Services Trust, 2008.

19 Hawton K, Harriss L, Hall S, Simkin S, Bale E, Bond A. Deliberate self-harm in Oxford, 1990-2000: a time of change in patient characteristics. Psychol Med 2003; 33: 987-95.

20 World Health Organization. The ICD-10 Classification of Mental and Behavioural Disorders: Clinical Descriptions and Diagnostic Guidelines. WHO, 1992.

21 Linsley KR, Schapira K, Kelly TP. Open verdict v. suicide - importance to research. Br J Psychiatry 2001; 178: 465-8.

22 Waern M, Rubenowitz E, Allebeck P, Runeson B, Skoog I, Wilhelmsson K. Risk factors for suicide in 'young old' and 'very old'. Acta Psychiatr Scand 2002; 105: 160.

23 World Health Organization. Definition of an Older or Elderly Person. WHO, 2011 (http://www.who.int/healthinfo/survey/ageingdefnolder/en/index.html).

24 Hawton $\mathrm{K}$, Harriss L. The changing gender ratio in occurrence of deliberate self-harm across the lifecycle. Crisis 2008; 29: 4-10.
25 Pierce D. Deliberate self-harm in the elderly. Int J Geriatr Psychiatry 1987; 2: $105-10$.

26 Frierson RL. Suicide attempts by the old and very old. Arch Intern Med 1991; 151: $141-4$.

27 Nowers M. Deliberate self-harm in the elderly - a survey of one London borough. Int J Geriatr Psychiatry 1993; 8: 609-14.

28 Dombrovski AY, Szanto K, Duberstein P, Conner KR, Houck PR, Conwell Y. Sex differences in correlates of suicide attempt lethality in late life. Am J Geriatr Psychiatry 2008; 16: 905-13.

29 Kapur N, Cooper J, King-Hele S, Webb R, Lawlor M, Rodway C, et al. The repetition of suicidal behavior: a multicenter cohort study. J Clin Psychiatry 2006; 67: 1599-609.

30 Owens D, Horrocks J, House A. Fatal and non-fatal repetition of self-harm: systematic review. Br J Psychiatry 2002; 181: 193-9.

31 Dennis $M$, Wakefield $P$, Molloy $C$, Andrews $H$, Friedman T. Self-harm in older people with depression: comparison of social factors, life events and symptoms. Br J Psychiatry 2005; 186: 538-9.

32 O'Connell H, Chin AV, Cunningham C, Lawlor BA. Recent developments: suicide in older people. BMJ 2004; 329: 895-9.

33 Runeson B, Tidemalm D, Dahlin M, Lichtenstein P, Langstrom N. Method of attempted suicide as predictor of subsequent successful suicide: national long term cohort study. BMJ 2010; doi: 10.1136/bmj.c3222.

34 National Collaborating Centre for Mental Health. Self-Harm: The Short-Term Physical and Psychological Management and Secondary Prevention of SelfHarm in Primary and Secondary Care. Gaskell/British Psychological Society, 2004.

35 Royal College of Psychiatrists. Assessment Following Self-Harm in Adults College Report CR122. Royal College of Psychiatrists, 2004. 